•综述・

\title{
遥感技术在鸟类生态学研究中的应用
}

\author{
雷 倩 1,2 李金亚 ${ }^{1}$ 马克明 $1^{*}$ \\ 1 (中国科学院生态环境研究中心城市与区域生态国家重点实验室, 北京 100085) \\ 2 (中国科学院大学, 北京 100049 )
}

\begin{abstract}
摘要: 获取鸟类活动及生境信息是鸟类生态学研究的基础, 而遥感技术弥补了传统野外调查方法的缺陷, 提供了 获取多种信息的新途径。应用遥感技术的鸟类生态学研究热点从最初的种群行为观察, 到栖息地选择, 再到生境 适宜性、破碎化及人为干扰探究等, 随着技术的不断发展也在扩展和变化。不同波段或组合下的遥感技术各有所 长。光学遥感应用广泛, 尤其是信息量较大的红外波段图像和作为野外鸟巢及物种活动监测常用工具的红外相机; 多光谱图像常用于栖息地制图以及地物识别, 高空间分辨率的数据甚至可对鸟类种群进行直接计数; 高光谱数据 则可对光谱特征相似的地物进行更为精确的区分和反演; 激光雷达遥感主要用于栖息地植被结构的三维探测, 为 了解鸟类栖息地选择提供更好的依据。微波遥感在飞鸟探测上应用颇多, 近年来多极化数据在复杂栖息地精确制 图上也具有优势, 但成本较高、解译复杂且推广度较低。在实际应用中, 遥感数据时空尺度的选择会影响研究结 果, 部分遥感反演参数也缺乏生态学意义。多源遥感数据的结合应用能够提升制图分类的精度, 实现数据的时空 分辨率互补, 优化鸟类生态研究所需参数。未来的遥感技术在鸟类生态学中的应用应致力于提供更加明确的光谱 信息、相对简便的解译方法, 以及更为合理的多源数据组合方式等。
\end{abstract}

关键词：微波遥感; 红外; LiDAR; 多光谱; 高光谱; 生境反演; 地物识别

\section{Applications of remote sensing technology in avian ecology}

\author{
Qian Lei ${ }^{1,2}$, Jinya $\mathrm{Li}^{1}$, Keming Ma ${ }^{1 *}$ \\ 1 State Key Laboratory of Urban and Regional Ecology, Research Center for Eco-Environmental Sciences, Chinese \\ Academy of Sciences, Beijing 100085 \\ 2 University of Chinese Academy of Sciences, Beijing 100049
}

\begin{abstract}
Avian ecological studies tend to center on birds and their habitats. According to the literature, studies in avian ecology have shifted from focusing on behavior and habitat selection to focusing on human disturbance, habitat suitability and habitat structure, which has been made possible partially due to remote sensing (RS) technology. Characteristics and applications of RS data are varied. Here, we assessed various RS methods, considering the current state of avian ecology. Light remote sensing is most commonly used. Infrared trigger cameras and video complement field work to monitor brooding, defensive and other behaviors, while the infrared images contain massive amounts of data. Multi-spectral images are used most frequently for mapping habitat and can directly track species when captured at a high spatial resolution. Hyperspectral data has great potential in classifying objects with similar spectral characteristics. LiDAR data mainly contributes to studies of habitat structure. Researchers have used Radar to monitor flying birds over extended periods of time, where the microwave images with multi-polarization may promote the precision of mapping complex habitats. In practice, we recognize that data scale may affect study results and that some RS inversion model parameters lack ecological significance. Multi-source data could enhance mapping accuracy and provide context for the intersection of spatial and temporal resolutions of images. In the future, RS technology development should pay more attention to provide specific spectral information, more convenient interpretation methods, and more rational multi-source data combinaions, for a better use of them.
\end{abstract}

Key words: microwave remote sensing; infrared; LiDAR; multi-spectral; hyperspectral; habitat parameter inversion; object identification

收稿日期: 2018-05-15; 接受日期: 2018-08-14

基金项目：国家重点研发计划项目(2017YFC0505800, 2016YFC0500406)和国家自然科学基金(41601439)

* 通讯作者 Author for correspondence. E-mail: mkm@rcees.ac.cn 
鸟类种群数量、动态、行为特征以及栖息地质 量、空间分布等基础信息的获取是鸟类生态学研究 的基础。传统鸟类监测多依赖于人为定点观测, 对 于活动范围较大或夜间活动的鸟类等局限性较大, 且耗时耗力, 计数及定标均容易产生主观偏差; 在 栖息地方面, 样线调查是植被情况、高程分布等数 据的主要来源, 但易受地形、天气等因素影响, 研 究的时空尺度受限。而遥感技术具有数据来源多 样、信息量丰富、观测范围广并且信息时效性强等 特点, 正越来越多地被用于鸟类动态及其栖息地监 测研究中(Kerr et al, 2010; 王勇等, 2012; Pimm et al, 2014)。

依据电磁波波段分类组合, 遥感数据可分为微 波遥感、可见光遥感、红外遥感、多光谱及高光谱 遥感等(赵英时, 2013)。不同的遥感波段获取的信息 不尽相同(表1): 雷达数据可用于监测鸟类的飞行 活动, 部分高空间分辨率图像还能够进行鸟类种群 计数; 红外触发相机可对物种进行识别及活动监测; 而热红外成像则能够观察鸟类的夜间活动情况; 多 /高光谱遥感数据可通过波段组合, 利用地物反射 特征的差异, 通过解译分辨出不同类型的下垫面利 用情况，也可通过结合实测数据模拟反演、推断植 被、建筑物群、水体(Lyzenga, 1978; Legleiter et al, 2004)等的物理特征; 激光雷达遥感则在植被垂直
结构分析上有优势(Lefsky et al, 1999)。这些遥感数 据或是作为物种活动的依据和环境参数, 或是与地 面调查结合, 通过物种-生境关系模型等分析方法 进行鸟类生态学研究, 从而了解鸟类活动规律和评 估、预测鸟类适宜栖息地等。

基于Web of Science核心数据库，参考《遥感大 词典》(陈述彭，1990)，选取 “remote sensing*”、 “LiDAR”、“camera”、“multispectral”、“radar”、 “hyperspectral” 等相关检索词搜索遥感相关文献; 选取“bird*”、“avian*”、“aves”、“avifauna”、“tinamou” 等相关检索词搜索鸟类研究文献，得到遥感与鸟类 生态学文献数据库, 文献时间截至2017年12月31日。

由图1A可以看出, 利用遥感数据进行鸟类生 态学研究的文章数量逐年递增, 尤其是在 20 世纪 90 年代初及近几年增幅较大，表明遥感技术在鸟类生 态学研究中的重要性在不断上升。而图1B可表明遥 感数据类型在鸟类生态学研究中的应用分布趋势。 早期使用的遥感技术以雷达为主，后期常用多光谱 卫星图像、红外相机等, 近年来LiDAR数据也成为 一个重要的应用类型。

簰选文献关键词，以5年为时间间隔，分别进 行研究趋势的关键词热词分析。由图2可以看出，鸟 类生态学的研究早期多关注物种本身, 如种群识 别、行为观察(受食、筑巢、育雉)等, 以及定性化的

表1 不同波段/多波段遥感数据在鸟类生态学研究中的应用

Table 1 Applications of different band or multi-band remote sensing data in bird ecological research

\begin{tabular}{|c|c|c|c|}
\hline $\begin{array}{l}\text { 波段(组合)类型 } \\
\text { Available bands }\end{array}$ & $\begin{array}{l}\text { 波长 } \\
\text { Spectral range }\end{array}$ & $\begin{array}{l}\text { 可获取信息类型 } \\
\text { Available information }\end{array}$ & 相关文献 Reference \\
\hline $\begin{array}{l}\text { 可见光波段 } \\
\text { Visible light bands }\end{array}$ & $0.4-0.7 \mu \mathrm{m}$ & A, B, C, D, E, H & $\begin{array}{l}\text { Rodgers et al, 1995; Abd-Elrahman, 2005; Evans et al, 2009; Delord et al, 2015; } \\
\text { Chen \& Zhang, 2015; Christie et al, } 2016\end{array}$ \\
\hline $\begin{array}{l}\text { 红外遥感 } \\
\text { Infrared band }\end{array}$ & $0.7-14 \mu \mathrm{m}$ & A, B, C, D, F, I, K, L, M & $\begin{array}{l}\text { Avery \& Haines-Young, 1990; Hüppop et al, 2006; MartiCardona et al, 2008; Lagos } \\
\text { et al, 2008; Mayer et al, 2009; Zhao et al, 2013; Vanhellemont \& Ruddick, } 2014\end{array}$ \\
\hline $\begin{array}{l}\text { 多光谱遥感 } \\
\text { Multispectral }\end{array}$ & $0.4-14 \mu \mathrm{m}$ & $\begin{array}{l}\text { C, D, E, F, H, I, J, K, L, } \\
\text { M, N, O }\end{array}$ & $\begin{array}{l}\text { Schwaller et al, 1989; Osborne et al, 2010; Tøttrup et al, 2008; Daily et al, 2001; } \\
\text { Rodrigues et al, 2012; Tebbs et al, 2013; Dwyer et al, } 2013\end{array}$ \\
\hline $\begin{array}{l}\text { 高光谱遥感 } \\
\text { Hyperspectral }\end{array}$ & $0.4-14 \mu \mathrm{m}$ & $\mathrm{D}, \mathrm{F}, \mathrm{J}, \mathrm{K}, \mathrm{L}, \mathrm{M}$ & $\begin{array}{l}\text { Gong et al, 1997; Martin et al, 1998; Costa et al, 2007; Goodenough et al, 2012; } \\
\text { Brydegaard et al, } 2013\end{array}$ \\
\hline $\begin{array}{l}\text { 激光雷达 } \\
\text { LiDAR }\end{array}$ & $0.24-1 \mathrm{~mm}$ & E, G, N & $\begin{array}{l}\text { Lefsky et al, 2002; Bradbury et al, 2005; Shi \& Cao, 2007; Goetz et al, 2010; Guo et } \\
\text { al, } 2016\end{array}$ \\
\hline $\begin{array}{l}\text { 微波遥感 } \\
\text { Microwave (Radar) }\end{array}$ & $1-100 \mathrm{~cm}$ & $\mathrm{~B}, \mathrm{D}, \mathrm{E}, \mathrm{F}, \mathrm{I}, \mathrm{J}, \mathrm{M}, \mathrm{N}$ & $\begin{array}{l}\text { Diehl et al, 2003; Taft et al, 2003; Boerner et al, 2008; Dabrowska-Zielinska et al, } \\
\text { 2009; Pistolesi et al, 2015; Ruan et al, } 2017\end{array}$ \\
\hline
\end{tabular}

$\mathrm{A}$ : 育倠; B: 飞行活动; C: 地面活动; D: 个体识别/计数; E: 制图; F: 植被识别; G: 植被结构; H: 常用遥感指数; I: 明水面及植被下漫 水面; $\mathrm{J}$ : 水深; $\mathrm{K}$ : 温度; $\mathrm{L}$ : 水体浑浊度; $\mathrm{M}$ : 土壤湿度; $\mathrm{N}$ : 地形; $\mathrm{O}$ : 夜间灯光。

A, Brooding; B, Flight activity; C, Ground activity; D, Identification and individual counting; E, Mapping; F, Vegetation recognition; G, Vegetation structure; H, Remote sensing index; I, Water surface and water area with vegetation cover; J, Depth of water; K, Temperature; L, Water turbidity; M, Soil moisture; N, Topography; O, Night-time light. 

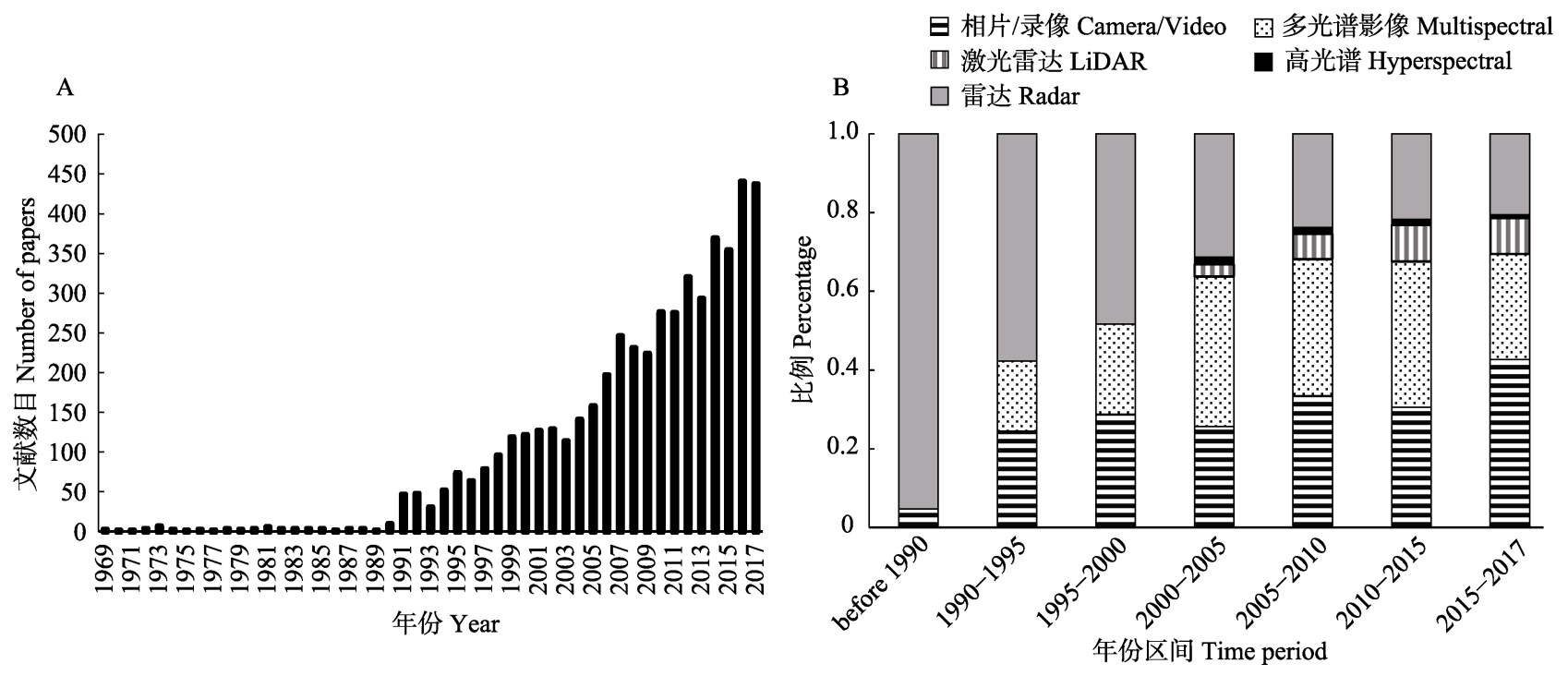

图1 利用遥感技术进行鸟类生态学研究的文章数目 $(\mathrm{A})$ 及不同年份区间的鸟类生态学研究中不同遥感技术的应用占比(B)

Fig. 1 The yearly number of avian ecological researches with remote sensing (A) and the application proportion of different remote sensing technology in bird ecological study (B)
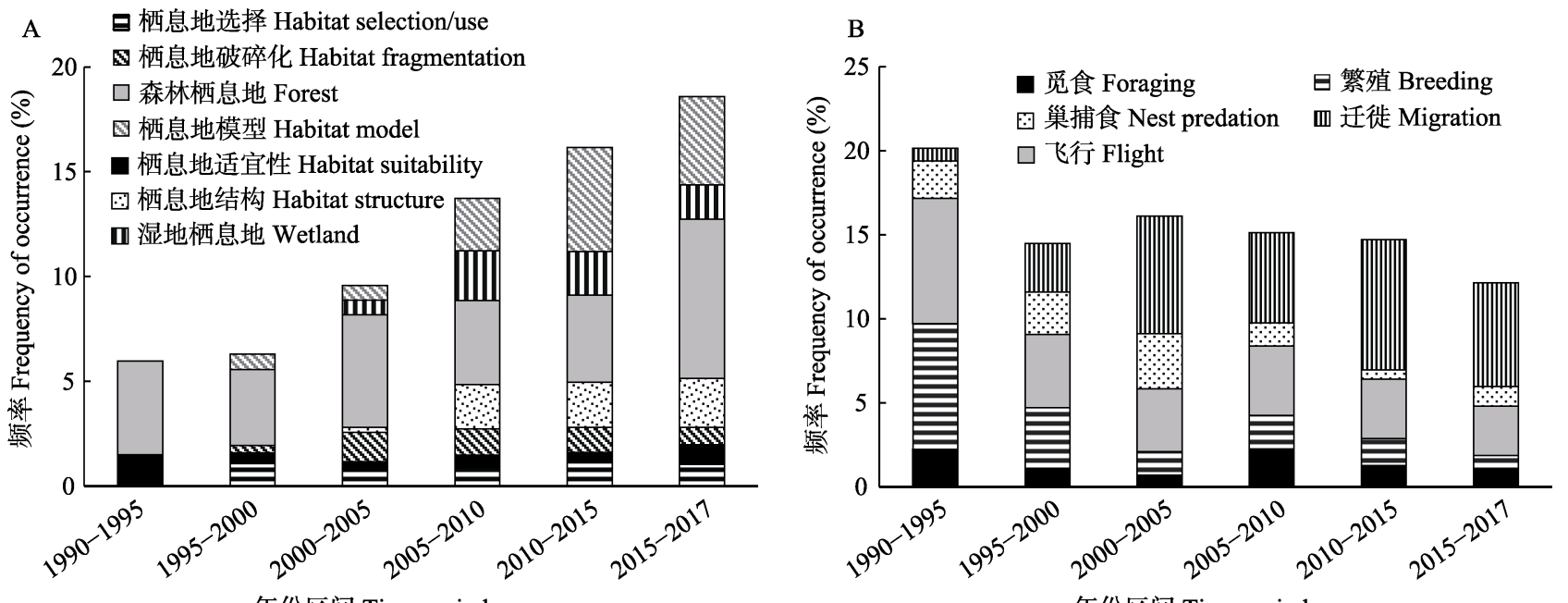

图2 基于Web of Science核心合集数据库的鸟类生态学研究中应用遥感技术的文献关键词热词分析。A：栖息地研究; B: 鸟 类行为相关研究。

Fig. 2 The key words analysis of avian ecological research with remote sensing according to the core database in Web of Science. A: Habitat study; B: Bird behavior study.

栖息地选择利用; 之后在鸟类食性、长距离迁徙、 人为保护管理等方面进行了拓展, 鸟类迁徙的研究 热度也在逐年提升; 近年来开始关注栖息地结构, 结合各项技术进行多尺度下的动态变化监测、栖息 地破碎化、适宜性评估、生物多样性保护以及人类 干扰影响(城市化、道路影响等)的分析等研究。尽 管年际间各研究方向的变化趋势并不明显, 但可以 看出, 基于遥感技术的鸟类生态学研究在坚持最初
研究内容的同时，也在不断地拓展，更加注重栖息 地与物种的联系、人为活动以及气候等因素的影响 和变化, 并逐渐发展定量模型进行探究。

不断发展的遥感技术在推动着研究的进步。本 文梳理了不同波段及组合下的遥感技术在鸟类生 态学研究中的应用, 分析了数据互补、融合应用的 潜力，以及在鸟类及其栖息地监测应用中的可能发 展方向。 


\section{光学遥感}

光学遥感是指从紫外到红外光学波段内获取 目标信息的一种遥感技术，依托于不同的搭载平台， 其波段组合以及时空分辨率都不尽相同, 按照光谱 波段组合类型大致可以分为以下几种: 可见光遥 感、红外遥感、多光谱遥感、高光谱遥感、激光雷 达遥感等。

\section{1 可见光遥感}

可见光遥感是基于可见光波段成像的遥测技 术, 单纯的可见光遥感主要以照相机、摄像机等为 载体。摄影数据的呈现方式是RGB彩图和视频, 数 据较为直观, 在物种观察以及栖息地测绘、动态监 测方面均有应用。

\subsection{1 物种/种群监测}

可见光图像可以直接辨识物种, 借助不同的搭 载平台如无人机、风笔等能够直接监测鸟类种群及 其筑巢情况(O’Connell et al, 2010; Weissensteiner et al, 2015; Delord et al, 2015)。例如Rodgers等(1995) 利用地面实地调查的林鹳(Mycteria american)巢数 结果也证实了航片调查的可信度。随着这类图像分 辨率的提高, 研究者们也尝试从半空中对一些个体 较大的水鸟进行跟踪调查和计数(Jones et al, 2006), 并不断改进算法以提高种群调查的准确度 (Abd-Elrahman, 2005)。由此可以看出可见光数据在 一定的空间分辨率下能够作为物种/种群识别的有 力工具。部分研究也会以摄影数据所得的物种活动 信息为基础, 分析人类活动对鸟类分布的影响情况 (Groom et al, 2013)。此外, 也需要注意工作状态的 设备在监测过程中对鸟类可能造成的干扰(Vas et al, 2015; Christie et al, 2016)。

\subsection{2 栖息地分类与制图}

摄影图像达到一定的空间分辨率之后，地表特 征也可以直观地区分, 能够用于栖息地制图及信息 提取，例如航片可作为城市绿地(Evans et al, 2009)、 林地灌丛生境、湖泊湿地等各类栖息地的制图数据 源。在栖息地的动态监测上, 时间灵活的航拍摄影 数据也有较好的应用潜力, 可监测水鸟栖息地滩 涂、水面变化等; 依据航拍历史档案可分析林鸟栖 息地的景观变化(Boren et al, 1999)。近年来也有利 用航片作为土地利用覆被的基础数据以确定鸟类 栖息地选择的研究(Jung et al, 2016)。

\subsection{3 栖息地特征参数反演}

可见光波段较少用于环境参数反演, 但也有研 究能够基于三波段进行遥感指数构建。如MODIS 数据中陆地波段结合一定的算法可对水体浑浊程 度进行监测，促进水鸟栖息地质量评估(Chen \& Zhang, 2015)。也有研究表明红绿蓝波段组合的植被 指数与传统的归一化植被指数 (normalized difference vegetation index, NDVI)作用类似，可用于小区 域范围内的植被信息提取(汪小钦等, 2015)。

\section{2 红外遥感}

红外遥感是利用红外波谱区波段进行探测的 遥感技术，包括用于探测地物热辐射特征的热红外 遥感，以及多光谱影像中的反射红外图像部分。前 者与摄影装置结合得到的红外相机是鸟类行为观 察、种类调查的常见工具，热红外成像能够在夜间 观察鸟类活动; 后者则更多地用于栖息地环境监 测、制图分类辅助等(图3)。

\subsection{1 物种/种群监测}

在鸟类个体及种群监测中, 红外相机和热红外 成像是观察鸟类特别是地栖型走禽的重要工具。其 中，红外触发锁定物种出现的时间，通过相机获取 的图片、视频，可以用于物种识别和行为观察; 热 红外图像不受日光限制，可以在夜间进行观测。多 数鸟类个体节律行为如迁徙(Hüppop et al, 2006)、受 食及筑巢等行为的研究很大程度上都基于该类技 术应用，这也是单红外波段遥感在鸟类生态学中最 主要的应用方式。Vanhellemont和Ruddick (2014)利 用热红外成像仪评估了风电场对飞行的鸟、蝙蝠等 生物的影响，而赵玉泽等(2013)利用红外触发相机 监测了白冠长尾雉(Syrmaticus reevesii)的季节活动 从而发现了雌雄个体不同季节的受食活动差异。同 理, 红外相机借助搭载平台还可对林鸟如栗冻 (Turdus rubrocanus)的巢防御行为进行监测(Hu et al, 2017)。此外，短波红外光谱也可用于特定目标监测, 如依据鸟巢的材质与卵在短波红外光谱的反射率 高的特性，探测隐蔽的鸟巢、卵等(Mayer et al, 2009)。

\subsection{2 栖息地分类与制图}

在栖息地地物信息提取上，基于辐射红外特性， 红外波段图像可以识别、监测水体及间接监测水体 中对温度变化敏感的物种, 助益水鸟及其栖息地的 研究。水体的光谱特征在近红外波段中与其他地物 
A

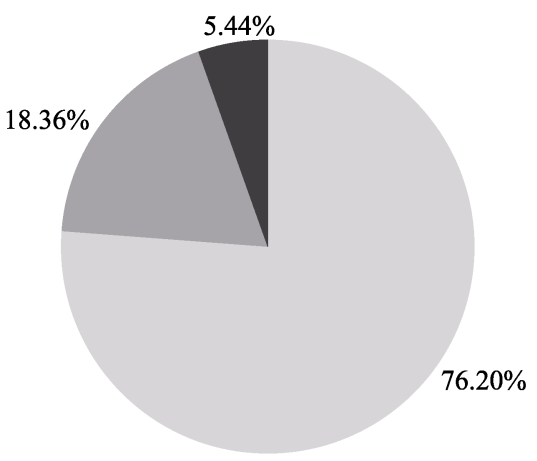

$\square$ 育雉、䍃食等行为观察 Brooding and foraging observation

$\square$ 栖息地制图分类 Habitat mapping and classification

其他 Others
B

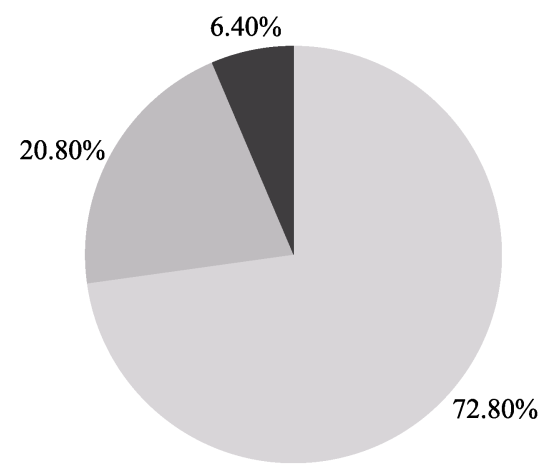

$\square$ 红外相机及热红外成像 Infrared trigger camera /video and infrared imager

$\square$ 近红外图像 Near-infrared image

中红外图像 Mid-infrared image

图3 红外波段在鸟类生态学中的应用情况(不包括多光谱)。A：应用领域; B: 应用波段。

Fig. 3 The proportion of application types of infrared image in bird ecological research. A, Application field; B, Application band.

差异较大, 遥感图像解译常借助近红外、短波红外 波段图层对水体信息进行识别(梅安新, 2001), 用于 滩涂、湖泊等水鸟栖息地的制图和动态监测。此外, 近红外波段图像的纹理特征也可用于栖息地对比 和区分, 例如Tuttle等(2006)利用ASTER高级空间热 辐射反射探测器的红、绿、近红外波段数据中的遥 感图像纹理(image texture), 来区分白、褐两种白喉 带卅(Zonotrichia albicollis)亚种在繁殖期间对栖息 地利用的差异。结果发现两者只在近红外波段的图 像中有区别。白色亚种的栖息地纹理均匀, 而褐色 亚种的栖息地图像频谱差异较大。除了栖息地差异, 生境破碎化也可以用近红外波段的图像进行甄别 (Fuller, 2001)。另外, Wood等(2012)研究表明也可利 用航拍的近红外图像纹理获取林地植被水平结构 信息, 为林鸟栖息地构成建模提供参数。

\subsection{3 栖息地特征参数反演}

在参数反演研究中, 基于红外波段的温度探测 可对水生植被情况进行间接监测, 从而获取水鸟食 物信息, 为鸟类迁徙重要停歇受食地监测研究提供 数据(Marticardona et al, 2008)。除了常见的结合可 见光波段等进行地物信息反演，单近红外波段的反 演前景也十分可观。如基于近红外波段数据得出的 土壤湿度信息可以作为苏格兰黑腹滨䂆岛(Calidris alpina) 种群数预测的重要参数 (Avery \& HainesYoung, 1990)。Lagos等(2008)利用近红外光谱信息 对黑颈天鹅(Cygnus melancoryphus)栖息的湿地水 体浑浊度的反演探测, 实现了水鸟栖息地水质的远 程动态监测。

\section{3 多光谱遥感}

多光谱遥感指的是具有两个以上波谱通道传 感器对地物进行同步成像的一种遥感技术，它将物 体反射辐射的电磁波信息分成若干波段进行接收 和记录。

\subsection{1 可见光-近红外组合的高空间分辨率遥感}

这一类型的遥感主要以较高的空间分辨率为 主要特征, 空间分辨率多优于 $10 \mathrm{~m}$, 地物边界较为 清晰, 有利于通过形状、颜色、纹理等特征进行目 视判别, 如常见的QuickBird、IKonos、GeoEye以及 我国的高分二号等。

(1)物种/种群监测。在个体、种群监测方面, 与 高清摄影图像类似，高空间分辨率图像可以直接用 于种群计数与物种鉴别。如Sasamal等(2008)利用 Quickbird图像中水体与鸟类的明暗反射差异实现 了湖中火烈鸟(Phoeniconaias minor)种群的分布监 测。随着分辨率与算法的发展, 地表覆被较为复杂 的栖息地中的物种识别研究也进一步增多。Fretwell 等(2017a)基于 $0.3 \mathrm{~m}$ 空间分辨率的WorldView-3数据 实现了对南大西洋岛屿的信天翁(Diomedea exulans) 种群的数量估计。

(2)栖息地分类与制图。在栖息地制图及变化监 测上, 因其数据价格较高, 常用于地物结构复杂或 小范围的典型区域研究中。随着栖息地精细化制图 需求的增大, 高分影像也逐渐在栖息地分类与制图 (Zohmann et al, 2013)、监测及模拟等方面发挥作 用。Bolca等(2014)利用多时相的Quickbird图像对有 水鸟分布的河口三角洲土地利用变化进行监测。 
Haywood和Stone (2011)则是采用SPOT图像中对植 被、土壤湿度变化敏感的短波红外波段与地形数据 结合, 进行森林退化及破坏情况的高分制图及环境 因子变化监测, 降低对鸟类生存的威胁。栖息地中 的植被情况可直接影响鸟类生存, 高分数据影像因 其高时空分辨率特性近年来也在生境植被结构 (Broughton et al, 2012)、季相变化监测(Mexicano et al, 2013)等研究中发挥着作用。

\subsection{2 中低空间分辨率多光谱遥感}

相较于高分影像, 这一类型影像的空间分辨率 大多较低, 波段数相应较多, 能够提供更多的光谱 信息, 有利于通过光谱、颜色、纹理等特征进行一 定程度上的计算机自动识别, 以Landsat系列以及 MODIS最为典型。

(1)物种/种群监测。在鸟类个体/种群识别研究 中, 以Landsat系列卫星为代表的多光谱数据可用于 部分景观结构比较单一的地区如极地的鸟类群体 探查。Schwaller等(1989)利用Landsat TM图像评估 了阿德利企我(Pygoscelis adeliae)筑巢点的分布变 化, 后在南极洲海岸线上利用Landsat ETM+数据调 查了其种群的分布点及范围, 结果显示与航拍图像 调查结果有 $96 \%$ 的相似度, 并发现了 6 处之前并未 报道的地区(Schwaller et al, 2013)。也有些研究综合 利用Landsat影像对生境范围内鸟类粪便痕迹进行 识别, 揭示种群分散点(Fretwell \& Trathan, 2009); 或者进行以Landsat ETM+数据结合检索算法获取 种群丰富度的监测研究(Lynch \& Schwaller, 2014),
以Landsat 8 反演的亮度和地温进行帝企找的种群自 动监测统计(She et al, 2017)。但上述研究的下垫面 相对单调, 在温热带区域要利用图像直接评估鸟类 种群状况的困难较大。鸟类种群分布预测研究中, 更多地会利用栖息地数据建立与鸟类的联系，采用 模型拟合等方式进行间接预测统计。

(2)栖息地分类与制图。相较于鸟类种群计数与 活动监测, 多光谱影像更多的是用于栖息地制图及 变化研究(图4), 尤其是Landsat、MODIS等长时间序 列数据对于栖息地历史变化等监测具有十分重要 的意义, 相关研究热点也从单纯的栖息地食源利用 转至栖息地破碎化、垂直结构等方面。Landsat影像 可分辨出景观中森林覆被与人为活动区的面积变 化，探究林鸟栖息地的森林破碎化程度与其物种丰 富度的关系。Daily等(2001)指出集约型土地利用可 能会导致农村类型的潜在栖息地鸟类生物多样性 的降低。Shirley等(2013)通过TM图像波段组合发现 栖息地未分类的原始多光谱图像可辅助实现较高 的鸟类分布预测成功率，该研究第一次仅利用光谱 反射识别了多个物种的一般分布模式，一定程度上 解决了目标导向性土地分类模型在不同对象物种 中通用性较差的问题。多时相的NOAA气象卫星所 搭载的AVHRR传感器图像数据则可用作大空间尺 度下的栖息地类型判断, 有对长距离迁徙鸟类如大 鸨 (Otis tarda) 等分布预判的潜力 (Osborne et al, 2010)。MODIS数据则以其重访周期短、成本低等 特征在宏观环境因子变化监测中存在优势。 $36.12 \%$

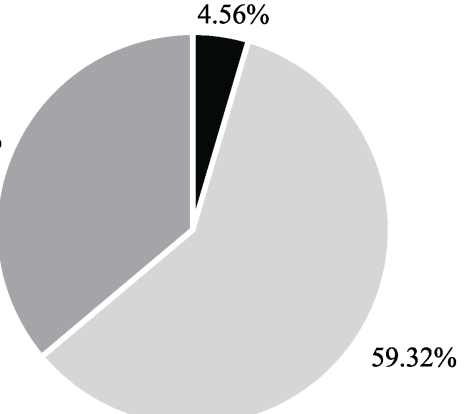

— 物种识别与监测 Species identification and observation 口 栖息地分类制图及变化监测 Habitat mapping and change monitoring 口 栖息地特征因子反演 Habitat characteristic factor inversion

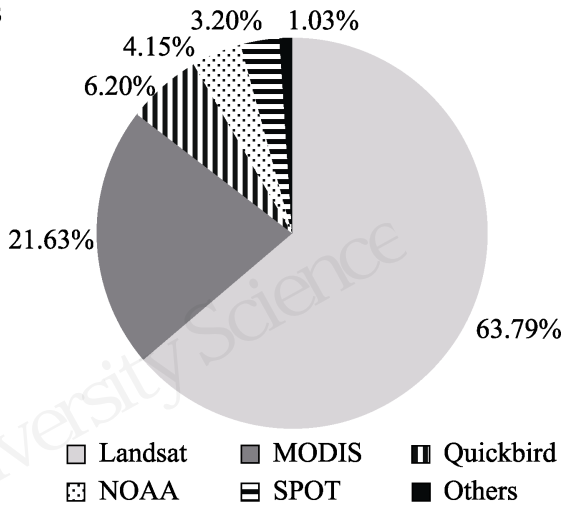

图4 多光谱数据在鸟类生态学研究中的应用方式(A)及常用遥感数据(B)比例分布

Fig. 4 The proportion of application types of multispectral image data in bird ecological research. A, Application field; B, Common remote sensing sensors. 
(3)栖息地特征参数反演。除了通过波段获取的 图像光谱、纹理差异了解栖息地情况外, 通过波段 组合运算, 构建一些特征指数, 以利于突出某一方 面特征, 有助于提取所需信息。多光谱图像数据的 蓝绿色波段 $(0.45-0.60 \mu \mathrm{m})$ 一般用于分辨植被，红 色波段(0.60-0.70 $\mu \mathrm{m})$ 对叶绿素的敏感度高, 近红 外波段信息量大, 所以多光谱图像在实际的解译过 程中多会采用红外波段与可见光的组合。例如Tebbs 等(2013)在肯尼亚地区的Lake Bogoria利用红和近 红波段遥感对叶绿素进行反演, 开发了蓝藻 (Cyanobacteria)的监测指标, 对火烈鸟的食物进行 监测, 为火烈鸟保护提供了有效信息。由红色可见 光波段与部分红外波段组合得到的NDVI是最常见 的衡量植被特征的指数。例如基于NOAA卫星数据 得到的NDVI指数可以反映鸟类迁徙路径上环境条 件食物丰富度, 得出区域的初级生产力, 与迁徙鸟 类的迁徙路线、停歇点等习性变化作对比, 探索候 鸟对环境变化的响应情况, 或者预测鸟类到达地区 的时间等(Gordo, 2007; Tøttrup et al, 2008)。Dodge 等(2014)指出基于MODIS下的NDVI数据, 欧洲天 气预报中心所得温度、风速数据, 飞行器模拟得到 的顺风、侧风及气流抬升速度等环境参数, 再结合 红头美洲鹑(Cathartes aura) 10年迁徙数据, 可描述 其迁徙行为响应环境变化的情况。此外, MODIS遥 感数据除了能够进行常用植被指数等生境因子的 反演, 其多时相的遥感数据还能够获取云层覆盖的 动态信息等气候因子，优化物种分布模型预测的结 果(Wilson \& Jetz，2016)。美国国防气象卫星 (DMSP/OLS)夜间灯光探测影像(近红-可见光组合) 也可以作为研究城市化夜光对鸟类影响的生境指 示参数, 如有研究表明部分海鸥(Larus canus)的迁 徙路线会被夜光干扰(Rodrigues et al, 2012), 但也

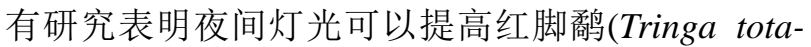
nus)夜间受食的能力(Dwyer et al, 2013)。

\section{4 高光谱遥感}

高光谱遥感是指在可见光至红外波段范围内, 用大量非常窄且连续的光谱通道对地物持续成像 的遥感技术。在鸟类生态学研究上主要应用于更为 精细的地物信息识别和反演(图5)。

\subsection{1 物种/种群监测}

在鸟类及其种群监测上, 高光谱数据凭借其较 高的光谱分辨率、极化分析可以得到雷达无法获取

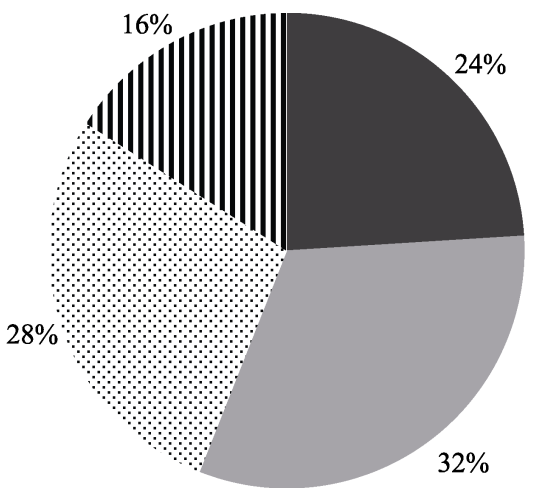

植被生理信息反演 Vegetation information inversion $\square$ 植被识别、区分 Vegetation identification and classification 提升土地利用分类精度 Accuracy improvement of land cover 【 鸟羽、粪渍等识别 Bird information identification

图5 高光谱数据在鸟类生态学研究中的应用类型分布 Fig. 5 The proportion of application types of hyperspectral data in bird ecological research

的物种信息。如Brydegaard等(2013)研究指出，红外 高光谱偏振成像可以通过羽毛的微特征结构与光 谱、极化效应的关联，达到识别高空、夜空鸟类物 种的目的。

\subsection{2 栖息地分类与制图}

较高的光谱分辨率使得高光谱遥感更有助于 地物识别和精细化分类。研究表明结合距离算法、 光谱角填图(spectral angle mapping, SAM)等分类方 法, 可以对森林、湿地等数十种植被进行识别(Gong et al, 1997; Costa et al, 2007), 高精度分类使得湿地 变化的监测效果也得到了提高。

\subsection{3 栖息地特征参数反演}

高光谱数据提供了更广的可选波段范围, 在地 物信息反演时，能够获取相关性更高、更为精确的 结果。例如一些植物生理特征和水文特征的精确监 测。Santos等(2010)利用高光谱数据对混合针叶林的 病株进行识别, 从而获取林木死亡率，作为红豚木 鸟(Picoides borealis)休毠繁殖的松林栖息地质量的 评估指标。Cui等(2013)测定了苔草属(Carex)植物叶 高光谱反射率以及其粗蛋白、磷含量，建立多元回 归模型，从而对鄱阳湖鸟类越冬期间的苔草类植被 质量进行光谱估计。此外, Karaman等(2015)基于高 光谱 4 个特定谱带对土耳其火烈鸟生境水面进行水 文周期监测, 借助归一化差异水体指数(normalized difference water index, NDWI), 确定旱季的水面覆 盖比湿季少约 $40 \mathrm{~km}^{2}$ 。与ETM数据比较, 高光谱遥 
感的分类精度可达到 97.5\% (Martin et al，1998; Goodenough et al, 2012)。

\section{5 激光雷达遥感(LiDAR)}

作为传统雷达与现代激光技术结合的主动遥 感技术产物, 激光雷达近年来逐渐成为研究技术的 热点, 其点云数据可在栖息地研究中得到地物三维 结构信息, 反演植被群落结构参数等, 借此实现生 境的精细化制图与结构信息的获取。近年来, 结合 无人机搭载平台, LiDAR逐渐成为栖息地三维结构 研究的有力工具。

高精度的地形信息以及生境垂直结构功能参 数的获取(Lefsky et al, 2002), 有利于提高物种分布 模型预测精度，改善不同尺度下物种动态格局模拟 的精度及可靠性(Zellweger et al, 2013; 郭庆华等, 2016)。对林鸟而言，森林栖息地植被结构相对复杂， 研究不仅需要更为精确的土地覆被情况及变化的 数据，更依赖于LiDAR技术所能获取的植被结构信 息(Goetz et al, 2010)。对水鸟而言，激光雷达的点云 数据可以在滩涂测绘上与 $\mathrm{DEM}$ 数据结合, 从而获 取更为准确的滩涂地图(史照良和曹敏, 2007)。Hill 和Hinsley(2015)通过LiDAR所提供的植被结构数据, 建立了林鸟维鸟的体重与植被冠层高度的相关关 系, 得出在环境稳定的林地栖息地系统下, 两者呈 现稳定的正相关关系。

激光雷达所具备的反映栖息地三维结构的能 力对鸟类-栖息地关系模型的环境要素构建有很大 帮助。冠层及中层灌木结构信息、单株植物信息和 地形地表信息等均是栖息地模型中有利于提高预 测能力的参数。Goetz等(2014)基于LiDAR数据完善 了森林栖息地结构模型, 成功预测了鸟类的分布情 况。Bradbury等(2005)利用机载激光雷达数据分辨 出了作物与田间的边界结构, 预测了农田景观中的 鸟类分布情况。Martinuzzi等(2009)采用LiDAR数据 对林下灌木层的结构以及鸟类的出现情况进行了 分析，完善了对林下灌木不均匀性的定量化描述， 以及对鸟类栖息分布的模型预测。Lesak等(2011)则 在利用激光雷达数据获取森林结构信息之后, 建模 预测了林鸟的丰富度。

\section{6 小结}

光学遥感各类不同的光谱波段/波段组合在物 种、栖息地监测中均有应用。由于不同波段特点相 异, 搭载平台也不同, 其数据优缺点各异。
可见光遥感图像波段较少，信息挖掘潜力低, 但其图像直观，成本相对较低，在鸟类种群直接观 察以及生态科普中均有一定的应用前景，近年来应 用热度也较高。基于相机平台, 热红外遥感成像以 及红外相机主要用于鸟类行为观察等方面，而近、 中红外波段的影像在下垫面探测、地物信息挖掘等 方面应用较多。

高分辨率可见光-红外组合遥感图像纹理细 致、空间分辨率高, 有利于鸟类计数、高精度制图 以及生境变化动态监测, 但价格昂贵, 多用于小尺 度或典型区域研究。而中低空分辨率的多光谱遥感 数据时空分辨率适宜、波段较多，不仅可以根据影 像的形态和纹理差异辨识地物, 还可以根据光谱特 征差异进行信息提取，增加了信息量及计算机信息 自动提取的可能。尤其是其成本低、时间序列长，应 用广泛、技术相对成熟等优势，有利于各尺度地物 识别及历史变化监测等研究。高光谱图像可通过深 度挖掘光谱特征来识别、分析地物, 实现模型参数 优化及地物精细化识别与分类, 在发现新参数及推 动定量探究上潜力较大。相较于一般光学遥感, LiDAR也存在一定程度的成本较高、解译难度大等 问题, 但却可以获得常规遥感图像无法获取的三维 结构(赵一鸣等, 2014)。

\section{微波遥感}

微波遥感是指通过接收微波辐射能量以及电 磁波束的回波信号，对物体进行探测、识别和分析 的遥感技术。应用较早, 后期随着光学遥感的增多 逐渐转为辅助工具, 但应用微波遥感进行鸟类生态 学研究的文献数量大体上依旧呈现一个逐年增加 的趋势; 而在应用占比上，微波遥感前期占比较高， 之后逐年递减，近年来则是趋于平缓(图6)。

\section{1 物种/种群监测}

通过微波雷达技术进行鸟类观测已有近 70 年 的历史，其空域搜索获取回波的特性可用于计算鸟 群飞行方向、速度以及长时间跟踪记录鸟类生活规 律，并且受干扰小、自动化程度高(Gauthreaux \& Belser, 2003)。在鸟类监测中, 一方面, 以飞行安全 为目的的机场鸟类识别是雷达遥感的重要应用。数 据显示，约61.4\%的鸟击事故都发生在黄昏或黎明 (陈唯实和李敬，2017), 而探鸟雷达技术全天候续 航、不受光照条件限制以及能见度干扰的优势使其 


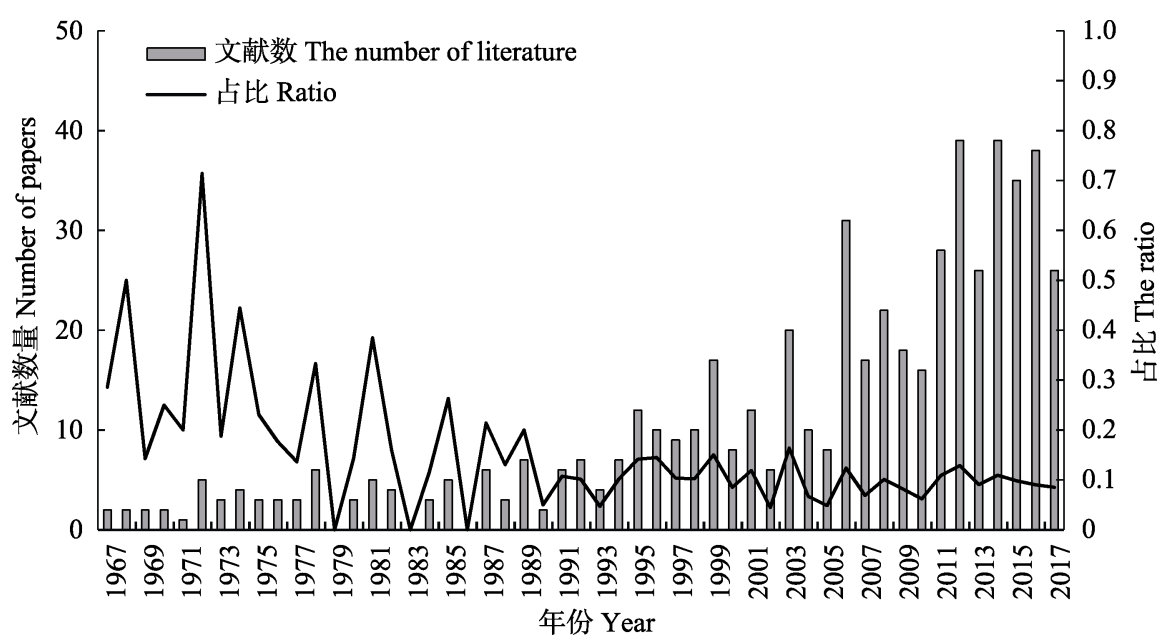

图6 历年微波遥感(雷达)在鸟类生态中的应用文献数, 以及历年在应用遥感技术的鸟类生态学文献中的历年占比

Fig. 6 The yearly quantity of publications of bird ecological study with radar and the proportion in of radar in all articles with remote sensing

成为观测的重要手段。Weber和Nohara(2011)指出基 于高度探测3D环绕式鸟类探测雷达系统可在机 场、飞行航线等空域中进行即时鸟类探查。另一方 面, 迁徙鸟群(特别是部分夜间迁徙类群)的动态监 测也是雷达遥感的应用领域, 如气象监测雷达可 以利用回波信号判别鸟类在迁徙过程中的飞行状 态(Wilson et al, 1994)。在偏振气象雷达及其他新算 法的协助下, 识别判断散射特征信号飞行目标的 精度也在逐渐提高(Alerstam et al, 2011; Farnsworth et al, 2016)。雷达还能够探测大规模迁徙鸟群的飞 行状态和栖息偏好。Diehl等(2003)利用WSR-88D天 气监测雷达在北美五大湖地区探究鸟群区域尺度 的迁徙模式, 发现部分鸟类黎明时分在大面积水域 上飞行时会提升飞行高度。Buler等(2012)则基于气 象雷达网络的反射率信息, 获取了越冬鸟群在栖息 地中的分布情况。此外, 鸟类在迁徙过程中的飞行 速度也可以借助雷达信号, 并结合空气动力学模型 进行测算(Bruderer \& Boldt, 2001; Alerstam et al, 2007)。

\section{2 栖息地分类与制图与特征信息提取}

在制图及地物信息提取上，微波遥感对地物介 电常数及表面粗䊁程度等特征变化有高敏感性, 可 用于鸟类栖息地环境研究。例如L波段下的ALOS (Advanced Land Observing Satellite) PALSAR合成 孔径雷达数据以及C波段下的RADARSAT卫星极 化数据, 既可用于高分辨率下鸟类林地沼泽栖息 地、迁徙停歇农田冬季景观等存在识别难度的栖息
地制图(Taft et al, 2003; Pistolesi et al, 2015)，也可用 于判断和预测水鸟栖息湿地的退化模式(Cornforth et al, 2013)。

微波遥感数据在植被下的水体识别、土壤湿度 监测等方面也有应用。郭殿繁等(2015)基于淹水植 被与非淹水植被的微波后向散射系数的明显差异, 利用ENVISAT ASAR合成孔径雷达图像识别了湿 地植被冠层下的淹水范围, 同时改善了光学影像分 类中的光谱异质现象, 减小了淹水植被分类错误概 率。Dabrowska-Zielinska等(2009)则利用ENVISAT ASAR、MERIS数据分别实现了湿地内土壤湿度与 生物质热通量等变化的监测。此外, 微波遥感也能 够获取植被高度、垂直结构等方面的信息，改良鸟 类栖息地适宜性模型及分布预测(Buermann et al, 2008)。同理，雷达图像数据也可对一些隐蔽性栖息 地(如浅色植被区、沙漠等类型的迁徙鸟类停歇地生 境)进行识别监测(Boerner \& Yamaguchi, 2008)。

\section{3 小结}

微波遥感不受光照条件限制、可全天候工作的 特征在飞行鸟类监测及栖息地环境物候等信息的 获取中有较大的优势。雷达探鸟技术相对成熟, 在 早期行为观察研究中应用颇广, 而成像雷达的发展 时间略短，且L、P、C、X等各类波段的探测优势有 所差异。作为测距衍生的微波遥感在变化监测上的 应用成效斐然, 但是这类工具主要还是侧重于探寻 地物辐射特性, 解译较为复杂, 识别物种等方面还 有待于解译算法的进步。展望未来, 微波遥感应在 
数据捕捉解译、可视化等方面进行简化及改良。

\section{3 展望}

鸟类生态学经过多年的探索积累, 在遥感技术 的辅助下，在鸟类行为、繁殖、栖息地等各方面均 有了较为详实的研究成果。未来的研究将会更加注 重鸟类生活史的动态监测、栖息地选择等多方面的 机理探讨。未来如何利用不同类型的传感器、不同 分辨率的遥感数据辅助实现鸟类生态学的研究, 进 而更加有效选择数据、挖掘所需信息, 提高信息准 确率及利用效率，仍需进一步探究。

\section{1 多源影像协同的优势}

不同遥感数据源特性不一, 适宜提取的信息也 不同(Clawges et al, 2008; Singh et al, 2017)。实际应 用中常结合研究所需(研究尺度、精度需求及信息可 获取性)及自身条件(数据可获取性、成本)等综合考 虑选择合适的数据源, 或是多类型数据源的组合, 以更好地提取目标对象特征。应用最广泛的多光谱 数据如Landsat、MODIS系列本质上也是可见光、红 外等多波段数据协同，在明晰各类波段所能区分的 地物类别后进行选取、组合，从而实现制图及生境 因子提取。而多光谱数据与LiDAR数据的联合, 以 及微波雷达极化数据的加入更是提高了栖息地因 子参数的数量及精度, 从而提高了生境评估的可靠 性与实用性。

多源数据融合也是提升精度的常用方式。例如 雷达图像与光学遥感数据结合识别地物信息可以 提高分类精度(Ruan et al, 2007)。除了增强成像式图 像的特征信息、锐化目标地物外，不同类型的传感 器也可以基于特定算法进行数据融合, 从而更好地 进行生境监测。Mirzaei等(2015)提出将红外相机、 船用雷达在贝叶斯模糊算法下进行多传感器数据 融合, 实现在风电场地区对迁徙的鸟类进行动态监 测, 为人为干扰下鸟类保护策略的制定提供依据。

\section{2 尺度效应}

生态学中, 尺度是维数、种类和组分三重概念 的结合, 包括现象或过程在时间、空间、生态学组 织层次的范围, 不同的现象、观测采样、分析等所 选取的时空范围、作用大小，以及景观中所涉及到 的空间粒度和时间间隔等(Wu et al, 2006; 张娜, 2006)。在遥感领域, 尺度更多地是指空间有效分辨 率单元和数据采集时间间隔(苏理宏等, 2001)。尺度
效应在鸟类生态学遥感技术应用中主要关注数据 获取的地表环境异质性，指的是不同尺度数据下同 一特征存在差异, 从而影响到研究的分析结果。在 定量遥感研究中, 为了得到合理的地物特征信息, 尺度效应探究越来越受到重视(李小文和王祎婷, 2013)。

鸟类栖息地地表覆被多以林地、水体及湿地等 边界模糊的地物类型为主。在鸟类栖息地选择和栖 息地适宜性评估中，遥感数据的尺度选择可直接影 响研究结果。在制图上, 相邻地物的边界混合像元 判断结果会影响适宜性栖息地的空间位置判断及 面积计算(孟斌和王劲峰, 2005)。此外，不同时空尺 度的遥感数据用于鸟类栖息地影响因子分析可得 到不同的结果。刘春悦等(2012)研究表明，基于二元 回归算法的繁殖栖息地选择关键影响因子分析结 果，会因遥感数据的空间尺度不同而不同。而 Suryan等(2012)指出，在探究预测海鸟分布热区时， 大时间尺度下的环境因子分析结果可优于短时间 尺度。所以在栖息地分析中, 基于研究目的选择合 适时空分辨率的遥感数据, 研究鸟类-生境多尺度 选择特征十分重要(Cushman \& Mcgarigal, 2002)。

\section{3 时效性}

在鸟类及其生境监测中, 某一时间段的遥感数 据所能提供的信息仅在一定时间内有价值，即存在 时效性。遥感数据成像时间与研究对象、研究过程 的一致性决定了监测结果的可靠性：一致性越高, 监测结果越可靠。鸟类在其生活史的不同阶段，其 行为与生境需求也不同。时间分辨率越高(重访周期 越短)、数据质量越好, 越有助于选取与建模数据时 间相一致的遥感数据, 进而越有利于提高监测结果 的可靠性(Ferraz et al, 2006)。

\section{4 反演参数的生态学意义}

现有的众多遥感反演指数因其研发时所对应 的生态、生理意义相对较为含糊, 并且受传感器类 型和影像时空分辨率不同的影响，在一定程度上缺 乏统一性及可对比性(Fensholt et al, 2009), 如被广 泛应用的归一化植被指数(NDVI)。同一遥感产品公 式在提出之初受实验条件、区域范围的限制，物理 机制难以类推。地物信息随着时间、地区的变化而 产生的差异性存在被忽视的可能, 导致得到的指数 和对应关系普适性较差。

遥感反演模型一般分为三类, 即经验模型(基 
于统计回归, 根据实地获取数据和光谱数据回归建 模)、半经验半分析模型和分析模型(根据地物反射 特性、光学参数等建立方程式)。经验模型是不同波 段组合计算后得到的遥感指数, 如反映植被特征的 红色植被指数 (red index, RI)、植被生产力指数 (vegetation productivity index, VPI)等。这类指数在 早期构建时均没有充分考虑大气、地面等的干扰。 半经验半分析模型是相对纯粹的物理模型, 参数算 法相对简单，应用更为广泛; 但其不确定性大, 对 应关系多样, 精度低, 在不同传感器类型数据中类 推性差(吴炳方和张沝, 2017)。分析模型基于物理方 程, 然而自然对象的系统复杂、未知量过多, 使得 方程式趋于无尽解, 引入先验数据后, 得到了大量 类似叶面积指数(leaf area index, LAI)的参数进行植 被冠层特征的描述; 但这类模型的解方程方式受限 于先验数据的准确度, 观测数据的微小变化也会引 起指数反演结果高度变异(Goel \& Thompson, 1984)。

单一传感器数据在光谱波段、时空分辨率、应 用成本上均有差异, 多源数据协同应用可以相互弥 补数据短板, 从而获取物种和栖息地中更多、更精 确的信息。在参数反演层面, 应用领域也应该多从 地物光谱特征出发, 充分考量各类传感器数据的成 本、波段信息特征、时效性、尺度效应等，而非直 接选取已有的遥感参数和指数进行生境条件的描 述和量化。

\section{$3.53 S$ 技术集成应用}

遥感(RS)、全球定位系统(GPS)和地理信息系统 (GIS)分别在地物信息获取、空间位置信息搜集、空 间数据分析管理输出等方面相辅相成, 是相关研究 的强大支撑。3S技术集成在鸟类生态学研究中应用 成效显著(江红星等, 2010)。

一般来说, 多以基于GPS的地面观测或者卫星 跟踪数据确定鸟类的栖息区域、偏好生境类型、繁 殖和行为特征; 利用遥感图像获取栖息地地表覆被 分类及生境因子; 再由GIS进行空间格局分析及栖 息地地物类别的评分、叠加分析等, 获取出现热点 范围及栖息地适宜性评估结果, 明晰候鸟迁徙方 向、路径及生境选择机理, 探究候鸟迁徙保护策略 以及人类活动或环境变化对鸟类种群及其栖息地 的影响(Higuchi et al, 2004)。多年的鸟类研究成果推 动了评估和预测模型的发展, Web of Science 所有 数据库中, 与鸟类生态学相关的物种栖息地模型及
栖息地适宜性模型研究文献数量在 460 篇左右, 21 世纪初增长幅度最为明显，其中 $60 \%$ 都在摘要或关 键词中提到了遥感技术。由此可见，遥感数据是其 最为常见的数据基础，3S技术不仅在量化生境质 量、监测物种分布等方面可发挥作用, 更是在优化 栖息地时空生境要素的数据搜集中，不断加强物种 一栖息地关系模型的应用能力, 提升了预测及评估 结果的精度。此外，3S技术还可为鸟类疾病防控提 供帮助(Gilbert et al, 2010)。

综上，遥感技术可以较全面地为鸟类生态学研 究提供物种、生境等信息，但其技术与应用发展程 度不对等。理论上能够做到的一些地物信息提取或 反演，应用中往往会受限于数据成本的高昂以及解 译过程的繁琐。此外, 从鸟类生态学家的角度看, 遥感技术更多在栖息地植被、水体等方面有识别作 用。常用的遥感图像如Landsat系列在植被垂直结构 及水体边界的区分上还有欠缺(基于常见解译、反演 方式)，微波及LiDAR技术的普及度也不足。所以在 未来研究中, 遥感学家与生态学家应加强交流合作, 在图像解译、反演和尺度推绎等层面构建一个映射 清晰的网络。更多地采用多数据协同、多源图像并 用，在尺度精度、时间迟滞性及栖息地结构等各方 面的综合考量下，选择最为适宜的传感器类型及遥 感平台图像，从而得到具备生态学意义的信息参 数。同时, 遥感图像解译也应向着全球标准化的方 向发展，以便于学者们交流和对比研究结果等。

\section{参考文献}

Abd-Elrahman A (2005) Development of pattern recognition algorithm for automatic bird detection from unmanned aerial vehicle imagery. Surveying and Land Information Science, 65, 37-45.

Alerstam T, Chapman JW, Backman J, Smith AD, Karlsson H, Nilsson C, Reynolds DR, Klaassen Raymond HG, Hill JK (2011) Convergent patterns of long-distance nocturnal migration in noctuid moths and passerine birds. Proceedings of the Royal Society B: Biological Sciences, 278, 3074-3080.

Alerstam T, Rosen M, Backman J, Ericson PGP, Hellgren O (2007) Flight speeds among bird species: Allometric and phylogenetic effects. PLoS Biology, 5, 1656-1662.

Avery MI, Haines-Young RH (1990) Population estimates for the dunlin Calidris alpina derived from remotely sensed satellite imagery of the Flow Country of northern Scotland. Nature, 344, 860-862.

Boerner WM, Yamaguchi Y (2008) Recent advances in 
POL-SAR \& POL-IN-SAR imaging of natural habitats and wetland remote sensing. In: Geoscience and Remote Sensing Symposium, pp. 293-294. IGARSS 2008, IEEE International.

Bolca M, Özen F, Güneş A (2014) Land use changes in Gediz Delta (Turkey) and their negative impacts on wetland habitats. Journal of Coastal Research, 30, 756-764.

Boren JC, Engle DM, Palmer MW, Masters RE, Criner T (1999) Land use change effects on breeding bird community composition. Journal of Range Management, 52, 420-430.

Bradbury RB, Hill RA, Mason DC, Hinsley SA, Wilson JD, Balzter H, Anderson GQA, Whittingham MJ, Davenport IJ, Bellamy PE (2005) Modelling relationships between birds and vegetation structure using airborne LiDAR data: A review with case studies from agricultural and woodland environments. Ibis, 147, 443-452.

Broughton RK, Hill RA, Freeman SN, Bellamy PE, Hinsley SA (2012) Describing habitat occupation by woodland birds with territory mapping and remotely sensed data: An example using the marsh tit (Poecile palustris). Condor, 114, 812-822.

Bruderer B, Boldt A (2001) Flight characteristics of birds: Radar measurements of speeds. Ibis, 143, 178-204.

Brydegaard M, Samuelsson P, Kudenov MW, Svanberg S (2013) On the exploitation of mid-infrared iridescence of plumage for remote classification of nocturnal migrating birds. Applied Spectroscopy, 67, 477-490.

Buermann W, Saatchi S, Smith TB, Zutta BR, Chaves JA, Mila B, Graham CH (2008) Predicting species distributions across the Amazonian and Andean regions using remote sensing data. Journal of Biogeography, 35, 1160-1176.

Buler JJ, Randall LA, Fleskes JP, Barrow WC, Bogart T, Kluver D (2012) Mapping wintering waterfowl distributions using weather surveillance radar. PLoS ONE, 7.

Chen SG, Zhang TL (2015) Evaluation of a QAA-based algorithm using MODIS land bands data for retrieval of IOPs in the eastern China Seas. Optics Express, 23, 13953-13971.

Chen SP (1990) Dictionary of Remote Sensing. Science Press, Beijing. [陈述彭 (1990) 遥感大词典. 科学出版社, 北 京.]

Chen WS, Li J (2011) Review on development and applications of avian radar technology. Modern Radar, 39, 7-17. (in Chinese with English abstract) [陈唯实, 李敬 (2017) 雷达 探鸟技术发展与应用综述. 现代雷达, 39, 7-17.]

Christie KS, Gilbert SL, Brown CL, Hatfield M, Hanson L (2016) Unmanned aircraft systems in wildlife research: Current and future applications of a transformative technology. Frontiers in Ecology and the Environment, 14, 241-251.

Clawges R, Vierling K, Vierling L, Rowell E (2008) The use of airborne LiDAR to assess avian species diversity, density, and occurrence in a pine/aspen forest. Remote Sensing of Environment, 112, 2064-2073.
Cornforth WA, Fatoyinbo TE, Freemantle TP, Pettorelli N (2013) Advanced land observing satellite phased array type L-band SAR (ALOS PALSAR) to inform the conservation of mangroves: Sundarbans as a case study. Remote Sensing, 5, 224-237.

Costa M, Araujo LE, Shaw A, Steckler C, Hill P (2007) Hyperspectral imagery for mapping intertidal vegetation at Roberts Bank tidal flats, British Columbia, Canada. Canadian Journal of Remote Sensing, 33, 130-141.

Cui LJ, Fei T, Qi Q, Liu YL, Wu GF (2013) Estimating Carex quality with laboratory-based hyperspectral measurements. International Journal of Remote Sensing, 34, 1866-1878.

Cushman SA, Mcgarigal K (2002) Hierarchical, multi-scale decomposition of species-environment relationships. Landscape Ecology, 17, 637-646.

Dabrowska-Zielinska K, Gruszczynska M, Lewinski S, Hoscilo A, Bojanowski J (2009) Application of remote and in situ information to the management of wetlands in Poland. Journal of Environmental Management, 90, 2261-2269.

Daily GC, Ehrlich PR, Sanchez-Azofeifa GA (2001) Countryside biogeography: Use of human-dominated habitats by the avifauna of southern Costa Rica. Ecological Applications, 11, 1-13.

Delord K, Roudaut G, Guinet C, Barbraud C, Bertrand S, Weimerskirch H (2015) Kite aerial photography: A low-cost method for monitoring seabird colonies. Journal of Field Ornithology, 86, 173-179.

Diehl RH, Larkin RP, Black JE (2003) Radar observations of bird migration over the Great Lakes. Auk, 120, 278-290.

Dodge S, Bohrer G, Bildstein K, Davidson SC, Weinzierl R, Bechard MJ, Barber D, Kays R, Brandes D, Han J (2014) Environmental drivers of variability in the movement ecology of turkey vultures (Cathartes aura) in North and South America. Philosophical Transactions of the Royal Society of London, 369, 20130195.

Dwyer RG, Bearhop S, Campbell HA, Bryant DM (2013) Shedding light on light: Benefits of anthropogenic illumination to a nocturnally foraging shorebird. Journal of Animal Ecology, 82, 478-485.

Evans KL, Newson SE, Gaston KJ (2009) Habitat influences on urban avian assemblages. Ibis, 151, 19-39.

Farnsworth A, Van-Doren BM, Hochachka WM, Sheldon D, Winner K, Irvine J, Geevarghese J, Kelling S (2016) A characterization of autumn nocturnal migration detected by weather surveillance radars in the northeastern USA. Ecological Applications, 26, 752-770.

Ferraz SFD, Capao L, Vettorazzi CA (2006) Temporal scale and spatial resolution effects on Amazon forest fragmentation assessment in Rondonia. International Journal of Remote Sensing, 27, 459-472.

Fensholt R, Rasmussen K, Nielsen TT, Mbow C (2009) Evaluation of earth observation based long term vegetation trends - Intercomparing NDVI time series trend analysis 
consistency of Sahel from AVHRR GIMMS, Terra MODIS and SPOT VGT data. Remote Sensing of Environment, 113, 1886- 1898.

Fretwell PT, Scofield P, Phillips RA (2017) Using super-high resolution satellite imagery to census threatened albatrosses. Ibis, 159, 481-490.

Fretwell PT, Trathan PN (2009) Penguins from space: Faecal stains reveal the location of Emperor penguin colonies. Global Ecology and Biogeography, 18, 543-552.

Fuller DO (2001) Forest fragmentation in Loudoun County, Virginia, USA evaluated with multitemporal Landsat imagery. Landscape Ecology, 16, 627-642.

Gauthreaux SA, Belser CG (2003) Radar ornithology and biological conservation. Auk, 120, 266-277.

Gilbert M, Newman SH, Takekawa JY, Loth L, Biradar C, Prosser DJ, Balachandran S, Rao MV, Mundkur T, Yan BP, Xing Z, Hou YS, Batbayar N, Natsagdorj T, Hogerwerf L, Slingenbergh J, Xiao XM (2010) Flying over an infected landscape: Distribution of highly pathogenic avian influenza H5V1 risk in south Asia and satellite tracking of wild waterfowl. Ecohealth, 7, 448-458.

Goel NS, Thompson RL (1984) Inversion of vegetation canopy reflectance models for estimating agronomic variables. 5 . Estimation of leaf-area index and average leaf angle using measured canopy reflectances. Remote Sensing of Environment, 16, 69-85.

Goetz SJ, Steinberg D, Betts MG, Holmes RT, Doran PJ, Dubayah R, Hofton M (2010) LiDAR remote sensing variables predict breeding habitat of a Neotropical migrant bird. Ecology, 91, 1569-1576.

Goetz SJ, Sun M, Zolkos S, Hansen A, Dubayah R (2014) The relative importance of climate and vegetation properties on patterns of North American breeding bird species richness. Environmental Research Letters, 9, 2033-2053.

Gong P, Pu R, Yu B (1997) Conifer species recognition: An exploratory analysis of in situ hyperspectral data. Remote sensing of Environment, 62, 189-200.

Goodenough DG, Chen H, Gordon P, Niemann KO, Quinn G (2012) Forest applications with hyperspectral imaging. In: 2012 IEEE International Geoscience and Remote Sensing Symposium, pp. 7309-7312. IEEE, Piscataway.

Gordo O (2007) Why are bird migration dates shifting? A review of weather and climate effects on avian migratory phenology. Climate Research, 35, 37-58.

Groom G, Stjernholm M, Nielsen RD, Fleetwood A, Petersen IK (2013) Remote sensing image data and automated analysis to describe marine bird distributions and abundances. Ecological Informatics, 14, 2-8.

Guo DF, Zang SY, Na XD (2015) Habitat suitability evaluation of red-crowned crane in Zhalong National Nature Reserve. Geography and Geo-Informaion Science, 31, 54-58. (in Chinese with English abstract) [郭殿繁, 藏淑英, 那晓东 (2015) 扎龙保护区丹顶鹤栖息地适宜性评价. 地理与地
理信息科学, 31, 54-58.]

Guo QH, Liu J, Li YM, Zhai QP, Wang YC, Wu FF, Hu TY, Wan HW, Liu HM, Shen WM (2016) Perspectives and prospects of unmanned aerial vehicle in remote sensing monitoring of biodiversity. Biodiversity Science, 24, 12491266. (in Chinese with English abstract) [郭庆华, 刘瑾, 李 玉美, 翟秋萍, 王永财, 吴芳芳, 胡天宇, 万华伟, 刘慧 明, 申文明 (2016) 生物多样性近地面遥感监测: 应用现 状与前景展望. 生物多样性, 24, 1249-1266.]

Haywood A, Stone C (2011) Mapping eucalypt forest susceptible to dieback associated with bell miners (Manorina melanophys) using laser scanning, SPOT 5 and ancillary topographical data. Ecological Modelling, 222, 1174-1184.

Higuchi H, Pierre JP, Krever V, Andronov V, Fujita G, Ozaki K (2004) Using a remote technology in conservation: Satellite tracking white-naped cranes in Russia and Asia. Conservation Biology, 18, 136-147.

Hill RA, Hinsley SA (2015) Airborne ldar for woodland habitat quality monitoring: Exploring the significance of LiDAR data characteristics when modelling organism-habitat relationships. Remote Sensing, 7, 3446-3466.

Hüppop O, Dierschke J, Exo KM, Fredrich E, Hill R (2006) Bird migration studies and potential collision risk with offshore wind turbines. Ibis, 148, 90-109.

Hu YB, Zhao QS, Lou YQ, Chen LJ, Antonio GM, Sun YH (2017) Parental attendance of Chestnut Thrush reduces nest predation during the incubation period: Compensation for low nest concealment? Journal of Ornithology, 158, 11111117.

Jiang HX, Liu CY, Hou YQ, Qian FW (2010) Application of RS, GIS and GPS techniques in study of avian habitat. Scientia Silvae Sinicae, 46, 155-163. (in Chinese with English abstract) [江红星, 刘春悦, 侯韵秋, 钱法文 (2010) 3S技 术在鸟类栖息地研究中的应用. 林业科学, 46, 155-163.]

Jones GP, Pearlstine LG, Percival HF (2006) An assessment of small unmanned aerial vehicles for wildlife research. Wildlife Society Bulletin, 34, 750-758.

Jung JF, Combs DL, Sowl KM (2016) Habitat selection by bristle-thighed curlews (Numenius tahitiensis) breeding within the Southern Nulato Hills, Alaska. Wilson Journal of Ornithology, 128, 727-737.

Karaman M, Budakoglu M, Avci DU, Ozelkan E, Bulbul A, Civas M, Tasdelen S (2015) Determination of seasonal changes in wetlands using CHRIS/Proba hyperspectral satellite images: A case study from Acigol (Denizli), Turkey. Journal of Environmental Biology, 36, 73-83.

Kerr JT, Isabelle D (2010) Habitat loss and the limits to endangered species recovery. Ecology Letters, 7, 1163-1169.

Lagos NA, Paolini P, Jaramilo E, Lovengree C, Duarte C, Contreras H (2008) Environmental processes, water quality degradation, and decline of waterbird populations in the Rio Cruces Wetland, Chile. Wetlands, 28, 938-950.

Lefsky MA, Cohen WB, Acker SA, Parker GG, Spies TA, 
Harding D (1999) LiDAR remote sensing of the canopy structure and biophysical properties of Douglas-fir western hemlock forests. Remote Sensing of Environment, 70, 339361.

Lefsky MA, Cohen WB, Parker GG, Harding DJ (2002) LiDAR remote sensing for ecosystem studies. Bioscience, 52, 19-30.

Legleiter CJ, Roberts DA, Marcus WA, Fonstad MA (2004) Passive optical remote sensing of river channel morphology and in-stream habitat: Physical basis and feasibility. Remote Sensing of Environment, 93, 493-510.

Lesak AA, Radeloff VC, Hawbaker TJ, Pidgeon AM, Gobakken T, Contrucci K (2011) Modeling forest songbird species richness using LiDAR-derived measures of forest structure. Remote Sensing of Environment, 115, 2823-2835.

Li XW, Wang YT (2013) Prospects on future developments of quantitative remote sensing. Acta Geographica Sinica, 68, 1163-1169. (in Chinese with English abstract) [李小文, 王 祎婷(2013) 定量遥感尺度效应刍议. 地理学报, 68, 1163-1169.]

Liu CY, Jiang HX, Zhang SQ, Hou YQ, Lu J (2012) Breeding habitat characteristics of red-crowned crane at Zhalong of Northeast China: A multi-scale approach based on TM and ASAR image data. Chinese Journal of Applied Ecology, 23, 491-498. ( in Chinese with English abstract) [刘春悦, 江红 星, 张树清, 侯韵秋, 陆军 (2012) 基于TM与ASAR遥感 数据的扎龙丹顶鹤繁殖栖息地多尺度特征. 应用生态学 报, 23, 491-498.]

Lynch HJ, Schwaller MR (2014) Mapping the abundance and distribution of Adelie penguins using Landsat-7: First steps towards an integrated multi-sensor pipeline for tracking populations at the continental scale. PLoS ONE, 9, 11, e113301.

Lyzenga DR (1978) Passive remote sensing techniques for mapping water depth and bottom features. Applied Optics, 17, 379-383.

Marticardona B, Steissberg TE, Schladow SG, Hook SJ (2008) Relating fish kills to upwellings and wind patterns in the Salton Sea. Hydrobiologia, 604, 85-95.

Martin ME, Newman SD, Aber JD, Congalton RG (1998) Determining forest species composition using high spectral resolution remote sensing data. Remote Sensing of Environment, 65, 249-254.

Martinuzzi S, Vierling LA, Gould WA, Falkowski MJ, Evans JS, Hudak AT, Vierling KT (2009) Mapping snags and understory shrubs for a LiDAR-based assessment of wildlife habitat suitability. Remote Sensing of Environment, 113, 2533-2546.

Mayer PM, Smith LM, Ford RG, Watterson DC, McCutchen MD, Ryan MR (2009) Nest construction by a ground- nesting bird represents a potential trade-off between egg crypticity and thermoregulation. Oecologia, 159, 893-901.

Mexicano L, Nagler PL, Zamora-Arrroyo F, Glenn EP (2013)
Vegetation dynamics in response to water inflow rates and fire in a brackish Typha domingensis Pers. marsh in the delta of the Colorado River, Mexico. Ecological Engineering, 59, 167-175.

Mei AX (2001) Remote Sensing Introduction. Higher Education Press, Beijing. (in Chinese) [梅安新 (2001) 遥感导论. 高等教育出版社, 北京.]

Meng B, Wang JF (2005) A Review on the methodology of scaling with geo-data. Acta Geographica Sinica, 60, 277288. (in Chinese with English abstract) [孟斌, 王劲峰 (2005) 地理数据尺度转换方法研究进展. 地理学报, 60, 277-288.]

Mirzaei G, Jamali MM, Ross J, Gorsevski PV, Bingman VP (2015) Data fusion of acoustics, infrared, and marine radar for avian study. IEEE Sensors Journal, 15, 6625-6632.

O’Connell AF, Nichols JD, Karanth KU (2010) Camera Traps in Animal Ecology: Methods and Analyses. Springer Science and Business Media, New York.

Osborne PE, Alonso JC, Bryant RG (2010) Modelling landscape-scale habitat use using GIS and remote sensing: A case study with great bustards. Journal of Applied Ecology, 38, 458-471.

Pimm SL, Jenkins CN, Abell R, Brooks TM, Gittleman JL, Joppa LN, Raven PH, Roberts CM, Sexton JO (2014) The biodiversity of species and their rates of extinction, distribution, and protection. Science, 344, 1246752.

Pistolesi LI, Ni-Meister W, McDonald KC (2015) Mapping wetlands in the Hudson Highlands ecoregion with ALOS PALSAR: An effort to identify potential swamp forest habitat for golden-winged warblers. Wetlands Ecology and Management, 23, 95-112.

Pradervand JN, Dubuis A, Pellissier L, Guisan A, Randin C (2014) Very high resolution environmental predictors in species distribution models: Moving beyond topography? Progress in Physical Geography, 38, 79-96.

Robinson WD, Bowlin MS, Bisson I, Shamounbaranes J, Thorup K, Diehl RH (2010) Integrating concepts and technologies to advance the study of bird migration. Frontiers in Ecology and the Environment, 8, 354-361.

Rodgers JA, Linda SB, Nesbitt SA (1995) Comparing aerial estimates with ground counts of nests in wood stork colonies. Journal of Wildlife Management, 59, 656-666.

Rodrigues P, Aubrecht C, Gil A, Longcore T, Elvidge C (2012) Remote sensing to map influence of light pollution on Cory's shearwater São Miguel Island, Azores Archipelago. European Journal of Wildlife Research, 58, 147-155.

Ruan RZ, Feng XZ, She YJ (2007) Fusion of Radarsat SAR and ETM plus imagery for identification of fresh water wetland. In: Geoinformatics 2007: Remotely Sensed Data and Information (eds Ju W, Zhao S), 6752(5), 74. International Society for Optics and Photonics, Nanjing.

Santos MJ, Greenberg JA, Ustin SL (2010) Using hyperspectral remote sensing to detect and quantify southeastern pine 
senescence effects in red-cockaded woodpecker (Picoides borealis) habitat. Remote Sensing of Environment, 114, 1242-1250.

Sasamal SK, Chaudhury SB, Samal RN, Pattanaik AK (2008) QuickBird spots flamingos off Nalabana Island, Chilika Lake, India. International Journal of Remote Sensing, 29, 4865-4870.

Schwaller MR, Olson J, Charles E, Ma ZQ, Zhu ZL, Dahmer P (1989) A remote sensing analysis of Adelie penguin rookeries. Remote Sensing of Environment, 28, 199-206.

Schwaller MR, Southwell CJ, Emmerson LM (2013) Continental-scale mapping of Adélie penguin colonies from Landsat imagery. Remote Sensing of Environment, 139, 353-364.

She XY, Ke CQ, Miao X, Zhang X, Zhang J (2017) An automated method for the detection of emperor penguin colonies from Landsat 8 imagery. Remote Sensing Letters, 8, 596-605.

Shirley SM, Yang Z, Hutchinson RA, Alexander JD, Mcgarigal K, Betts MG (2013) Species distribution modelling for the people: Unclassified landsat TM imagery predicts bird occurrence at fine resolutions. Diversity and Distributions, 19, 855-866.

Shi ZL, Cao M (2007) A study of surveying and mapping of island and tidal flat based on LiDAR. Bulletin of Surveying and Mapping, (5), 49-53. (in Chinese) [史照良, 曹敏 (2007) 基于LiDAR技术的海岛礁、滩涂测绘研究. 测绘 通报, (5), 49-53.]

Singh M, Tokola T, Hou Z, Notarnicola C (2017) Remote sensing-based landscape indicators for the evaluation of threatened-bird habitats in a tropical forest. Ecology and Evolution, 7, 4552.

Su LH, Li XW, Huang YX (2001) An review on scale in remote sensing. Advanced in Earth Science, 16, 544-548. (in Chinese with English abstract) [苏理宏, 李小文, 黄裕霞 (2001) 遥感尺度问题研究进展. 地球科学进展, 16, 544548.]

Suryan R, Santora J, Sydeman W (2012) New approach for using remotely sensed chlorophyll $a$ to identify seabird hotspots. Marine Ecology Progress, 451, 213-225.

Taft OW, Haig SM, Kiilsgaard C (2003) Use of radar remote sensing (RADARSAT) to map winter wetland habitat for shorebirds in an agricultural landscape. Environmental Management, 32, 268-281.

Tebbs EJ, Remedios JJ, Avery ST, Harper DM (2013) Remote sensing the hydrological variability of Tanzania's Lake Natron, a vital Lesser Flamingo breeding site under threat. Ecohydrology and Hydrobiology, 13, 148-158.

Tøttrup AP, Thorup K, Rainio K, Yosef R, Lehikoinen E, Rahbek C (2008) Avian migrants adjust migration in response to environmental conditions en route. Biology Letters, 4, 685.

Tran A, Goutard F, Chamaillé L, Baghdadi N, Seen DL (2010)
Remote sensing and avian influenza: A review of image processing methods for extracting key variables affecting avian influenza virus survival in water from Earth Observation satellites. International Journal of Applied Earth Observation and Geoinformation, 12, 1-8.

Turner W, Spector S, Gardiner N, Fladeland M, Sterling E, Steininger M (2003) Remote sensing for biodiversity science and conservation. Trends in Ecology and Evolution, 18, 306-314.

Tuttle EM, Jensen RR, Formica VA, Gonser RA (2006) Using remote sensing image texture to study habitat use patterns: A case study using the polymorphic white-throated sparrow (Zonotrichia albicollis). Global Ecology and Biogeography, 15, 349-357.

Vanhellemont Q, Ruddick K (2014) Turbid wakes associated with offshore wind turbines observed with Landsat 8. Remote Sensing of Environment, 145, 105-115.

Vas E, Lescroel A, Duriez O, Boguszewski G, Gremillet D (2015) Approaching birds with drones: First experiments and ethical guidelines. Biology Letters, 11, 20140754.

Wang XQ, Wang MM, Wang SQ, Wu YD (2015) Extraction of vegetation information from visible unmanned aerialvehicle images. Transactions of the Chinese Society of Agricultural Engineering, 31,152-159. (in Chinese with English abstract) [汪小钦, 王苗苗, 王绍强, 吴云东 (2015) 基于可见光波 段无人机遥感的植被信息提取. 农业工程学报，31， 152-159.]

Wang Y, Zhang ZW, Zheng GM, Li JQ, Xu JL, Ma ZJ, Biancucci AL (2012) Ornithological research: Past twenty years and future perspectives in China. Biodiversity Science, 20, 119-137. (in Chinese with English abstract) [王勇, 张正旺, 郑光美, 李建强, 徐基良, 马志军, Biancucci AL (2012) 鸟类学研究: 过去二十年的回顾和对中国未来发展的建 议. 生物多样性, 20, 119-137.]

Weber PT, Nohara TJ (2011) Device and method for 3D height-finding avian radar. US. Patent No. 7, 864, 103. US Patent and Trademark Office, Washington, DC.

Weissensteiner MH, Poelstra JW, Wolf JBW (2015) Lowbudget ready-to-fly unmanned aerial vehicles: An effective tool for evaluating the nesting status of canopy-breeding bird species. Journal of Avian Biology, 46, 425-430.

Wilson AM, Jetz W (2016) Remotely sensed high-resolution global cloud dynamics for predicting ecosystem and biodiversity distributions. PLoS Biology, 14, e1002415.

Wilson JW, Weckwerth TM, Vivekanandan J, Wakimoto RM, Russell RW (1994) Boundary-layer clear-air radar echoes: Origin of echoes and accuracy of derived winds. Journal of Atmospheric and Oceanic Technology, 11, 1184-1206.

Wood EM, Pidgeon AM, Radeloff VC, Keuler NS (2012) Image texture as a remotely sensed measure of vegetation structure. Remote Sensing of Environment, 121, 516-526.

Wu BF, Zhang M (2017) Remote sensing: Oberservations to data products. Acta Geographica Sinica, 72, 2093-2111. (in 
Chinese with English abstract) [吴炳方, 张炎 (2017) 从遥 感观测数据到数据产品. 地理学报, 72, 2093-2111.]

Wu J, Jones KB, Li H, Loucks OL (2006) Scaling and uncertainty Analysis in Ecology. Springer, Dordrecht.

Zellweger F, Braunisch V, Baltensweiler A, Bollmann K (2013) Remotely sensed forest structural complexity predicts multi species occurrence at the landscape scale. Forest Ecology and Management, 307, 303-312.

Zhang N (2006) Scale issues in ecology: Concepts of scale and scale analysis. Acta Ecologica Sinica, 26, 2340-2355. (in Chinese with English abstract) [张娜 (2006) 生态学中的 尺度问题: 内涵与分析方法. 生态学报, 26, 2340-2355.]

Zhao YM, Li YH, Shang YN, Li J, Yu Y, Li LH (2014) Application and development direction of LiDAR. Journal of Telemetry, Tracking and Command, 35, 4-22. (in Chinese with English abstract) [赵一鸣, 李艳华, 商雅楠, 李静, 于勇, 李凉海 (2014) 激光雷达的应用及发展趋势. 遥测遥控, 35, 4-22.]
Zhao YS (2013) Principle and Method of Remote Sensing Application Analysis. Science Press, Beijing. (in Chinese) [赵 英时 (2013) 遥感应用分析原理与方法. 科学出版社, 北 京.]

Zhao YZ, Wang ZC, Xu JL, Luo X, An LD (2013) Activity rhythm and behavioral time budgets of wild reeves's pheasant (Syrmaticus reevesii) using infrared camera. Acta Ecologica Sinica, 33, 6021-6027. (in Chinese with English abstract) [赵玉泽, 王志臣, 徐基良, 罗旭, 安丽丹 (2013) 利用红外照相技术分析野生白冠长尾雉活动节律及时间 分配. 生态学报, 33, 6021-6027.]

Zohmann M, Pennerstorfer J, Nopp-Mayr U (2013) Modelling habitat suitability for alpine rock ptarmigan (Lagopus muta helvetica) combining object-based classification of IKONOS imagery and Habitat Suitability Index modelling. Ecological Modelling, 254, 22-32.

(责任编委：王勇 责任编辑: 周玉荣) 